\title{
Obstructive Jaundice Caused by Primary Duodenal Lymphoma
}

\author{
Case Report and Review of the Literature
}

\author{
Ulrich Peter ${ }^{a}$ Hanspeter Honegger ${ }^{b}$ Hans Rudolf Koelz ${ }^{a}$ \\ ${ }^{a}$ Division of Gastroenterology and ${ }^{b}$ Clinic of Medical Oncology, Triemli Hospital, Zürich, Switzerland
}

\section{Dear Sir,}

Primary duodenal lymphoma is a rare neoplasm, and initial presentation with obstructive jaundice is even rarer. Primary B-cell lymphoma of the duodenum was shown to be the cause of biliary obstruction in the present case.

A 70-year-old woman was referred to our hospital in July 2005 because of pruritic jaundice and dyspepsia for a few days. There was no weight loss, fever or nocturnal sweats. The patient had a history of bronchoalveolar adenocarcinoma (pT1pN0) for which she had had a resection of the left lower pulmonary lobe 4 years ago. Physical examination on admission was normal apart from jaundice. Abnormal laboratory findings included bilirubin $154 \mu \mathrm{mol} / \mathrm{l}$ (normal value $<20$ ), alkaline phosphatase $547 \mathrm{IU} / 1(<117)$, and ALT $284 \mathrm{IU} / \mathrm{l}(<31)$; LDH was normal. CT scan showed dilated biliary and pancreatic ducts and a slight thickening of the duodenal wall without pancreatic or peripancreatic mass. Endoscopic retrograde cholangiography revealed an ulcerative tumor in the descending part of the duodenum including the papilla of Vater. There was massive dilatation of the biliary and pancreatic ducts with a stenotic section of about $3 \mathrm{~cm}$ in length in the distal common bile duct. A sphincterotomy was performed, and two 7Fr plastic double pigtail stents were placed and later replaced by a single $10-\mathrm{mm}$ selfexpanding metal stent. Unexpectedly, his- tology of the tumor showed diffuse large B-cell lymphoma. The patient was treated with six courses of the CHOP scheme of chemotherapy, accompanied by immunotherapy with rituximab and followed by consolidating radiotherapy. In the CT scan, a good remission of the duodenal wall thickening could be documented, and the patient has been well for 18 months since the first course of chemotherapy.

\section{Discussion}

Gastrointestinal lymphomas account for approximately $40 \%$ of all extranodal lymphomas and for $4-12 \%$ of all nonHodgkin lymphomas [1-3]. The incidence has been reported to be $0.8-1.2$ per 100,000 persons per year [4]. By far the most common site of primary gastrointestinal lymphoma is the stomach, accounting for 75$80 \%$ of all primary gastrointestinal lymphomas, followed by the ileum (12-15\%) and colorectum (approx. 5\%). The duodenum only accounts for $0.8-2 \%$ of all gastrointestinal lymphomas $[1,5]$.

Symptoms most often associated with primary lymphoma of the small intestine include abdominal pain, anorexia, weight loss and manifestations of bowel obstruction. Less often observed are nausea, vomiting or diarrhea and only rarely gastrointestinal bleeding, and obstructive jaundice is extremely rare $[5,6]$. Up until now 14 cases have been reported in the literature (table 1). Only 4 of them had symptoms suggesting lymphoma including fever and nocturnal sweats, while most had a history of weight loss probably due to local effects of the tumor.

Treatment in our case consisted of reestablishing biliary drainage by endoscopic retrograde cholangiography in order to relieve symptoms and to enable chemotherapy, followed by radiotherapy. In most previous cases, biliary drainage was established before starting chemotherapy. To the contrary, Dudgeon and Brower [7] reported 5 patients with obstructive jaundice due to non-Hodgkin's lymphoma who had chemotherapy using attenuated doses of doxorubicin and vincristine without prior biliary drainage, and jaundice disappeared in all. Although based on a small group of cases, the prognosis appears to be relatively favorable as shown in table 1 . Of 14 cases found in the literature, 7 were reported to be in complete remission during a mean follow-up of 19 months.

In conclusion, obstructive jaundice caused by primary duodenal lymphoma is a very rare event. In view of the minor risks of biopsy and the important consequences in case of finding a treatable disease like lymphoma, histological clarification should be attempted whenever feasible.

$\begin{array}{ll}\text { KARGER } & \odot \text { 2007 S. Karger AG, Basel } \\ \begin{array}{l}\text { Fax }+41613061234 \\ \begin{array}{l}\text { E-Mail karger@karger.ch } \\ \text { www.karger.com }\end{array}\end{array} & \begin{array}{l}\text { Accessible online at: } \\ \text { www.karger.com/dig }\end{array}\end{array}$

\author{
Hans R. Koelz, MD \\ Division of Gastroenterology, Department of Internal Medicine, Triemli Hospital \\ Birmensdorferstrasse 497 \\ CH-8063 Zürich (Switzerland) \\ Tel. +41 44466 1317, Fax +41 44466 2602, E-Mail hansrudolf.koelz@triemli.stzh.ch
}


Table 1. Primary duodenal lymphoma with obstructive jaundice

\begin{tabular}{|c|c|c|c|c|c|c|c|c|c|c|c|}
\hline Year & Country & Localization & Age & Sex & Symptoms & Histology & $\begin{array}{l}\text { Nonsurgical } \\
\text { biliary drainage }\end{array}$ & Surgery & $\begin{array}{l}\mathrm{CT} / \\
\mathrm{RT}\end{array}$ & $\begin{array}{l}\text { Follow- } \\
\text { up }\end{array}$ & Ref. \\
\hline 1986 & Kuwait & duodenum & 24 & $\mathrm{f}$ & $\begin{array}{l}\text { pain, nausea, } \\
\text { weight loss }\end{array}$ & poorly differentiated & & & RT & $\begin{array}{l}\text { exitus } \\
3 \text { months }\end{array}$ & 8 \\
\hline 1986 & $\begin{array}{l}\text { Kuwait } \\
\text { (Egyptian) }\end{array}$ & $\begin{array}{l}\text { stomach/ } \\
\text { duodenum }\end{array}$ & 30 & $\mathrm{~m}$ & $\begin{array}{l}\text { pain, weight } \\
\text { loss }\end{array}$ & $\begin{array}{l}\text { poorly differentiated } \\
\text { lymphocytic }\end{array}$ & PTCD & & RT & lost & 8 \\
\hline 1986 & USA & duodenum & 72 & $\mathrm{~m}$ & $\begin{array}{l}\text { anorexia, } \\
\text { weight loss }\end{array}$ & diffuse histiocytic & PTCD & & $\mathrm{CT}$ & $\begin{array}{l}\text { exitus } \\
2 \text { months }\end{array}$ & 9 \\
\hline 1987 & $\begin{array}{l}\text { South } \\
\text { Africa }\end{array}$ & duodenum & 56 & $\mathrm{~m}$ & $\begin{array}{l}\text { pain, anorexia, } \\
\text { weight loss }\end{array}$ & large cell (Western variant) & & Roux-en-Y & CT & lost & 10 \\
\hline 1993 & USA & duodenum & 35 & $\mathrm{~m}$ & bleeding & poorly differentiated & & & CT & $\begin{array}{l}\text { CR } \\
36 \text { months }\end{array}$ & 7 \\
\hline 1994 & Australia & papilla & 57 & $f$ & nausea & MALT & & Whipple & & $\begin{array}{l}\text { CR } \\
26 \text { months }\end{array}$ & 11 \\
\hline 1995 & USA & duodenum & 42 & $\mathrm{~m}$ & $\begin{array}{l}\text { pain, weight } \\
\text { loss, pancreatitis }\end{array}$ & large cell & ERCP & & $\mathrm{CT}$ & $\begin{array}{l}\text { CR } \\
21 \text { months }\end{array}$ & 12 \\
\hline 1997 & USA & papilla & 56 & $f$ & & follicle center & ERCP & Whipple & RT & $\begin{array}{l}\text { CR } \\
5 \text { months } \\
\end{array}$ & 13 \\
\hline 2001 & USA & papilla & 67 & $\mathrm{f}$ & weight loss & T-cell & ERCP & Whipple & & $\begin{array}{l}\text { exitus } \\
13 \text { months }\end{array}$ & 14 \\
\hline 2003 & Morocco & duodenum & 10 & $\mathrm{~m}$ & nausea & Burkitt & & & CT & $\begin{array}{l}\text { CR } \\
1 \text { month }\end{array}$ & 15 \\
\hline 2004 & $\begin{array}{l}\text { Switzer- } \\
\text { land }\end{array}$ & papilla & 54 & $\mathrm{~m}$ & $\begin{array}{l}\text { anorexia, } \\
\text { weight loss }\end{array}$ & T-cell & PTCD & & CT & $\begin{array}{l}\text { exitus } \\
2 \text { months } \\
\end{array}$ & 16 \\
\hline 2005 & Turkey & duodenum & 33 & $\mathrm{~m}$ & pain & intermediate grade B-cell & & $\begin{array}{l}\text { gastroentero- } \\
\text { and choledo- } \\
\text { choenterostomy }\end{array}$ & CT & $\begin{array}{l}\text { CR } \\
12 \text { months }\end{array}$ & 2 \\
\hline 2005 & Turkey & duodenum & 38 & $\mathrm{~m}$ & itching & diffuse B-cell & & & CT & exitus & 2 \\
\hline 2006 & Turkey & duodenum & 16 & $f$ & pain & Burkitt & & & CT & $\begin{array}{l}\text { CR } \\
36 \text { months }\end{array}$ & 17 \\
\hline
\end{tabular}

$\mathrm{CT}=$ Chemotherapy $\mathrm{RT}=$ radiotherapy $\mathrm{CR}=$ complete remission .

\section{References}

1 Cirillo M, Federico M, Curci G, Tamborrino E, Piccinini L, Silingardi V: Primary gastrointestinal lymphoma: a clinicopathological study of 58 cases. Haematologica 1992;77: 156-161.

2 Yildirim N, Öksüzoglu B, Budakoglu B, Vural $\mathrm{M}$, Abal H, Uncu D, Zengin N: Primary duodenal diffuse large cell non-Hodgkin lymphoma with involvement of ampulla of Vater: report of 3 cases. Hematology 2005;10:371-374.

3 Rosenberg SA, Diamond HD, Jaslowitz B, Craver LF: Lymphosarcoma: a review of 1,269 cases. Medicine (Baltimore) 1961;40:31-84.

4 Koch P, Del Valle F, Berdel WE, et al: Primary gastrointestinal non-Hodgkin's lymphoma. I. Anatomic and histologic distribution, clinical features, and survival data of 371 patients registered in the German Multicenter Study GIT NHL 01/92. J Clin Oncol 2001;18:3861-3873.

5 Domizio P, Owen RA, Shepherd NA, Talbot IC, Norton AJ: Primary lymphoma of the small intestine. A clinicopathological study of 119 cases. Am J Surg Pathol 1993;17:429442.

6 Boddie A, Eisenberg BL, Mullins JD, Schlichtemeier AL: The diagnosis and treatment of obstructive jaundice secondary to malignant lymphoma: a problem in multidisciplinary management. J Surg Oncol 1980;14: 111-123.

7 Dudgeon DJ, Brower M: Primary chemotherapy for obstructive jaundice caused by intermediate-grade non-Hodgkin lymphoma Cancer 1993;71:2813-2816.

8 Radhakrishnan S, Al Nakib B, Al Liddawi H, Al Ruwaih A: Primary gastrointestinal lymphoma complicated by common bile duct obstruction: report of two cases. Am J Gastroenterol 1986;81:691-694.

9 Barek L, Orron D: Non-Hodgkin's lymphoma presenting as periampullary mass with obstructive jaundice. J Comput Tomogr 1986;10 89-92.

10 Halline A, Lerios M, Melissas J, Segal I, Grieve TP: Primary lymphoma of the small bowel with obstructive jaundice and pancreatitis. S Afr Med J 1987;72:61-62.

11 Pawade J, Lee CS, Ellis DW, Vellar ID, Rode J: Primary lymphoma of the ampulla of Vater. Cancer 1994;73:2083-2086.

12 Schoeppner HL, Wong DK, Bresalier RS: Primary small bowel lymphoma manifested as obstructive jaundice in a patient with AIDS. South Med J 1995;88:583-585.
13 Misdraji J, Fernandez del Castillo C, Ferry JA: Follicle center lymphoma of the ampulla of Vater presenting with jaundice. Am J Surg Pathol 1997;21:484-488.

14 Weinstock LB, Swanson PE, Bennett KJ, Van Amburg A, Wald SM, Shah NB: Jaundice caused by a clinically undetectable T-cell lymphoma infiltrating the sphincter of Oddi. Am J Gastroenterol 2001;96:3186-3189.

15 Chat L, Mahi M, Chellaoui M, BenamourAmmar $\mathrm{H}$ : Ictère cholestatique révélant un lymphome duodénal primitif. J Radiol 2003; 84:617-619.

16 Buess M, Steuerwald M, Wegmann W, Rothen M: Obstructive jaundice caused by enteropathy-associated T-cell lymphoma in a patient with celiac sprue. J Gastroenterol 2004;39: 1110-1113.

17 Ugur H, Tacyildiz N, Yavuz G, Unal E, Sayili A, Emir S, Kansu A, Kuloglu Z, Bahar K: Obstructive jaundice: an unusual initial manifestation of intra-abdominal non-Hodgkin lymphoma in a child. Pediatr Hematol Oncol 2006;23:87-90. 